https://www.journal-imab-bg.org

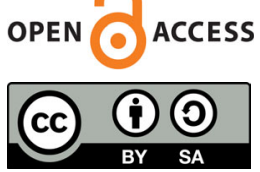

Case report

\title{
MIKULICZ'S SYNDROME ASSOCIATED WITH LEUKEMIA: A CASE REPORT
}

\author{
Elitsa Deliverska ${ }^{1}$, Lutchezar Stefanov ${ }^{1}$, Evgueniy Hadjiev², Zornitsa \\ Mihaylova $^{1}$, Dobrina Karayasheva ${ }^{3}$ \\ 1) Department of Oral and maxillofacial surgery, Faculty of Dental Medicine, \\ Medical University - Sofia, Bulgaria \\ 2) Department of Haematology, Medical Faculty, Medical University - Sofia, \\ Bulgaria \\ 3) Department of Conservative dentistry, Faculty of Dental Medicine, Medical \\ University - Sofia, Bulgaria
}

\begin{abstract}
Mikulicz's syndrome is a chronic swelling of the lacrimal and major salivary glands usually associated with significantly decreased or lack of lacrimation and xerostomia, accompanied by lymphocytic infiltration. It is associated with other entities like tuberculosis, lupus erythematosus, lymphoma, leukemia, etc.

A case of 70 years old man with painless, dense swelling of the left parotid gland and medical history of leukemia is presented. The decreased secretory activity of the parotid gland is observed. The patient is referred for laboratory blood test and ultrasound examination of the parotid glands. Mikulicz's syndrome associated with acute myelogenous leukemia was identified. Laboratory blood test reveals leukocytosis (118 g/L). The progress of the glands pathology seems to be associated with the severity of the main disease and appears to be a poor prognostic sign. Based on the findings the definitive diagnosis in the reported case is Mikulicz's syndrome associated with leukemia. The swelling is expected to disappear spontaneously or after treatment with corticosteroids. However, paliative treatment was conducted due to the severity of the systemic disease.
\end{abstract}

Keywords: Mikulicz's syndrome, salivary gland swelling, Extranodal lymphoid infiltrates,

\section{INTRODUCTION}

In 1888 Johann von Mikulicz-Radecki reported a case of 42-year-old man with a seven-month history of bilateral, painless, asymptomatic, and symmetrical swelling of the lacrimal, parotid, and submandibular glands. The lacrimal and salivary glands enlargement was associated with lymphocytic infiltrations upon microscopic examination. Massive round cell were observed with unchanged glandular acini, atrophy of the parenchyma and diffuse replacement by lymphoid tissue. The case was published in 1892, but cases of bilateral glandular swelling secondary to well-known pathology were also described in the article [1].
Schaffer and Jacobsen [2], in 1927 resolved the confusion by linking the case with other entities like tuberculosis, sarcoidosis, lymphoma, leukemia, etc. They divided the two pathologies, terming the idiopathic cases as Mikulicz's disease and cases associated with systemic disorder as Mikulicz's syndrome [2].

In 1933, Henrik Sjögren describes the clinical and histologic features in a newfound pathology associated with dry eyes, xerostomia, major salivary glands enlargement. The syndrome bears his name [3]. The lymphocytic infiltration and the relatively common clinical presentation has led to the acceptance of Mikulicz's syndrome and Sjögren syndrome to be considered as the same entity, and for many years the two terms have been used interchangeably. However, in the resent years the two syndromes have been distinguished [4]. It has been concluded that they differ in phenotypic, antibody and clinical findings. Mikulicz's syndrome has lower frequency of severe xerostomia, lacrimal gland dysfunction and does not lead to squamous metaplasia of the ocular surface as it is usually observed in Sjögren syndrome [4].

Sjögren syndrome, lymphomatous infiltration and Mikulicz's syndrome are entities included in the differential diagnosis of painless swelling of the lacrimal and salivary glands secondary to systemic disorder $[5,6,7,8]$. The most common systemic disorders accompanying Mikulicz's syndrome are leukemia [5], lymphomas [6], tuberculosis [7], sarcoidosis [8].

\section{AIM}

The aim of this article is to present a clinical case of 70-year-old male with the extremely rare Mikulicz's syndrome based on a medical history of acute myelogenous leukemia and left parotid gland enlargement with decreased salivation.

\section{CASE REPORT}

We present a 70-year-old male with swelling in the left parotid region (Fig. 1). Complaints of xerostomia were reported; vision and tearing were not affected. Night sweats 
and subfebrile temperature $\left(37.9^{\circ} \mathrm{C}\right)$ were reported. Significant facial asymmetry is observed. The left parotid gland is dense and solid but painless on palpation; the function is impaired with significantly reduced salivation. No abnormalities were found on palpation of the right parotid and the additional major salivary glands. General enlargement of the lymph nodes was noticed (cervical, axillary, inguinal). Laboratory blood tests reveal leukocytosis (18 $\mathrm{g} / \mathrm{L})$. The patient has a medical history of acute myelogenous leukemia, established nearly 20 months ago based on the following findings: erythrocyte sedimentation rate (ESR) $91 \mathrm{~mm} / \mathrm{h}$; Hb 82 g/L; Leuk. 7,3 G/L - Bl. 36\%; Thr. 159 G/L; Ac. ur. $169 \mu \mathrm{mol} / \mathrm{L}$; LDH 435 U/L; myelogram: normocellular bone marrow with $40 \%$ blast cells with characteristics of myeloblasts; $32 \%$ of myeloblasts have shown the following phenotype:

CD45low/CD34+/CD13+/CD117+/HLADR+/ CD 71+/CD38+/CD64+/CD11b+/MPO+/cyCD13+; overexpression of EVI 1 gene. Chemotherapy with Epirubicin, Mitoxantrone and Vinblastine have been prescribed as a palliative and substitution treatment. The myelogram 3 months ago shows normocellular bone marrow with $10 \%$ blast cells.

The patient was referred for ultrasound assessment. The examination does not reveal significant pathology in the glands because of the early stage (Fig. 2).

Palliative treatment was conducted due to the severity of the systemic disease.

Written informed consent was obtained from the patient to the inclusion of material pertaining to him.

Fig. 1. The Initial appearance of a patient with Mikulicz's Syndrome - facial asymmetry due to the enlargement of the left parotid gland is observed.

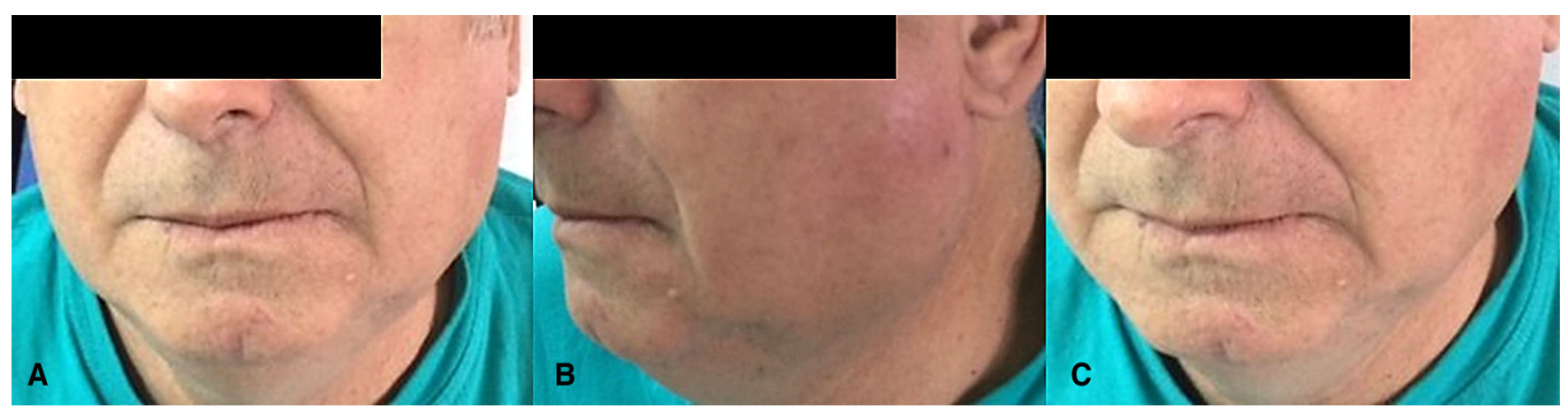

Fig. 2. Ultrasound examination of the parotid glands in a patient suffering from Mikulicz's Syndrome.

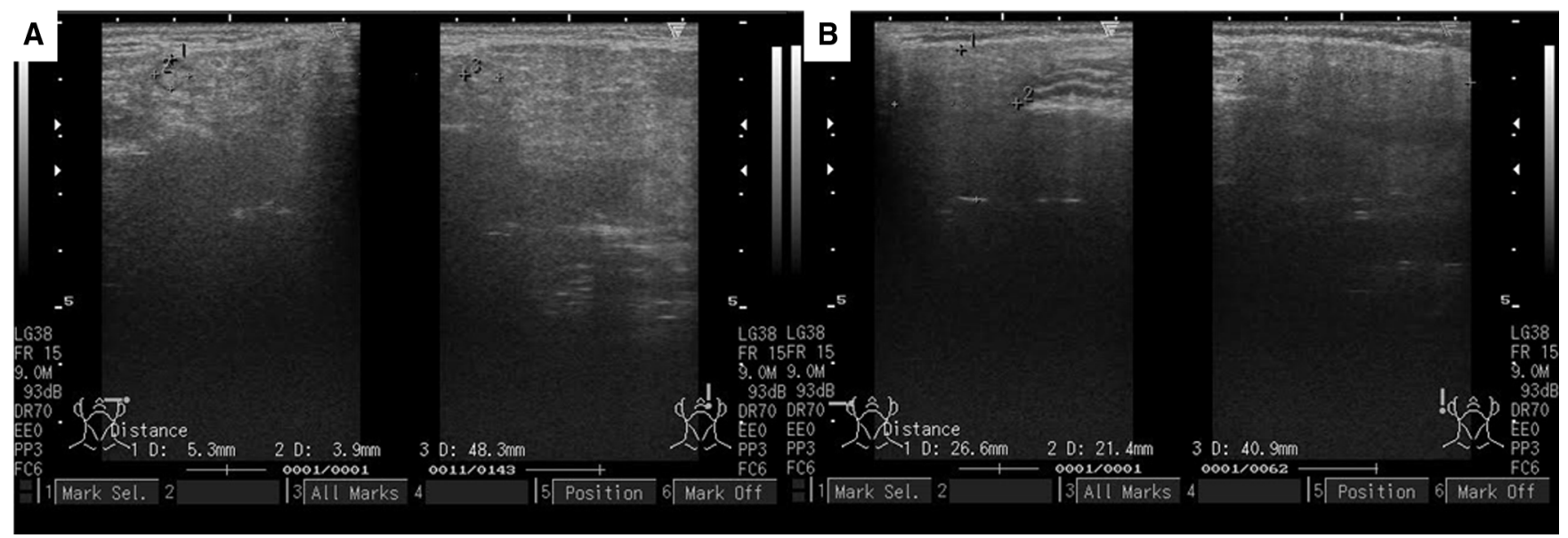

\section{DISCUSSION}

The heading Mikulicz's syndrome includes all the apparent enlargements of the lacrimal and salivary glands secondary to a pathologically defined disease. It is extremely rare pathology with lack of enough clinical cases described in the literature, especially in the past few years. The chief symptoms are associated with the pressure of the enlarged glands in combination with dry mouth and throat, difficulty in hearing, exophthalmos and vision disturbances. Usually, all the salivary and lacrimal glands are affected. However, a case has been reported where only the lacrimal glands are involved in the pathology of Mikulicz's syndrome [9].

One of the main entities commonly considered to be associated with Mikulicz's syndrome is leukemia [10]. Based on the clinical behavior, leukemia could be classified as acute or chronic; based on the primary hematopoietic cell line affected, leukemia is myeloid or lymphoid. Nearly $65 \%$ of patients with leukemia reviewed in the course of their disease oral signs and symptoms like gingival bleeding, petechiae, ecchymosis, gingival enlargement and ulceration followed my oral infection [11]. In ad- 
vanced cases, hyperplasia of lymphatic issues (i.e. Waldeyers ring, enlarged tonsils, etc.) may be noticed. Signs and symptoms resulting from leukemia are lymph nodes enlargement, enlargement of the spleen, liver. The most commonly affected lymph node groups are the cervical, axillary and inguinal lymph nodes. Anemia and fever may occur.

By the definitions in the literature, the diagnosis that we established falls in the category of Mikulicz's syndrome secondary to leukemia. The patient has notable facial asymmetry. No complaint of pain or severe xerostomia is presented. In the late stage, the entire gland is expected to be infiltrated and replaced, but the salivation will be maintained despite the infiltration of the parenchyma.

The white blood cell count is usually increased moderately: from 14000 to 35000 cells per cu. mm [12]. In our case, the white blood cell count is 18000 per cu. $\mathrm{mm}$. Sialography has been a suitable method to differentiate swelling arising from lymph node enlargement and those from salivary gland pathology. However, the contem- porary imaging methods are giving more reliable information about the patient's condition. Therefore, the ultrasound assessment applied in our study gave us appropriate information during the examination and confirmed the diagnosis.

The diagnosis Mikulicz's syndrome may be established according to the following points: 1. Painless, nontender usually bilateral swelling of the salivary and lacrimal glands; 2. The Rise in the white blood cell count; 3 . Enlargement of the liver and the spleen; 4. Generalized lymph node swelling [12]. In our case, most of the signs are presented.

\section{CONCLUSION}

Mikulicz's syndrome associated with leukemia is an extremely rare disease. The lymphocytic infiltration, as well as the salivary gland enlargement, is not life threatening complications. However, the clinical outcome depends on the severity of the systemic disease presented with leukemia in the reported case.

\section{REFERENCES:}

1. Mikulicz J. UE ber eine eigenartige symmetrische Erkrankung der Tranen und Mundspeicheldrusen. Beitr Chir Fortsch Gewidmet Theodor Billroth. Stuttgart, Germany: Enke; $1892 ; 610-30$

2. Schaffer AJ, Jacobsen AW. Mikulicz's syndrome: a report of ten cases. Am J Dis Child. 1927 Sept; 34(3):327-46. [CrossRef]

3. Morgan WS, Castleman B. A clinicopathologic study of Mikulicz's disease. Am J Pathol. 1953 MayJun;29(3):471-503. [PubMed]

4. Yamamoto M, Harada S, Ohara M, Suzuki C, Naishiro Y, Yamamoto H, et al. Clinical and pathological differences between Mikulicz's disease and
Sjogren's syndrome. Rheumatology (Oxford). 2005 Feb;44(2):227-34. [PubMed] [CrossRef]

5. Kaszewaska-Jablonska I. Mikulicz's syndrome in chronic lymphatic leukemia. Pol Arch Med Wewn. 1962; 32:367-72.

6. Kaufman SI. Mikulicz syndrome: an early manifestation of Hodgkin's disease. Eye Ear Nose Throat Mon. 1950 May; 29(5):257-8.

7. Garland HG, Thompson JG. Uveoparotid tuberculosis. QJM. 1933 Apr; 2(1):157-78.

8. Mahmod K, Khan A, Malik SA, Ilyas M. Mikulicz syndrome, an uncommon entity in Pakistan. $J$ Coll
Physicians Surg Pak. 2007 Feb; 17(2):101-2.

9. Kerschbaumer R. Ein Beitrag zur Kentniss der leukaemischen Erkrankung des Auges. Arch F Ophth. 1895 Nov; 41(3):99-122.

10. Jackson AS. Mikulicz's Disease. Am J Surg. 1945; 68(3):358-63.

11. Deliverska EG, Krasteva A. Oral signs of leukemia and dental management - literature data and case report. $J$ of IMAB. 2013 Oct-Dec; 19(4):388-91. [CrossRef]

12. Rowe SN. Mikulicz's Syndrome with Chronic Lymphatic Leukemia. New England J Med. 1930 Nov; 202(18):863-5.

Please cite this article as: Deliverska E, Stefanov L, Hadjiev E, Mihaylova Z, Karayasheva D. Mikulicz's syndrome associated with leukemia: a case report. J of IMAB. 2018 Apr-Jun;24(2):1995-1997.

DOI: https://doi.org/10.5272/jimab.2018242.1995

Address for correspondence:

Dr Zornitsa Mihaylova,

Department of Oral and maxillofacial surgery, Faculty of Dental medicine, Medical University - Sofia,

1, St. George Sofiyski str., 1431 Sofia, Bulgaria; tel.: +359 882492209

E-mail: zorrymihaylova@gmail.com 